\title{
Thermoelastic symmetric and antisymmetric wave modes with trigonometric functions in laminated plates
}

Kishori Lal Verma

\begin{abstract}
Background: Coupling term in coupled thermoelasticity generally is small for all materials and can be neglected. Neglecting the coupling term simplifies the analysis without noticeable effect on the frequency spectrum. In generalized theory of thermoelasticity, effect of increasing the relaxation time is to lower the speeds of the thermal modes. Since the effect of anisotropy of the material is quite pronounced on waves propagating in different directions along the plate. Thus, it is significant to consider the anisotropy of the material in order to accurately model the propagation characteristics.

Methods: A theoretical framework is developed for displacements and temperature expression of plane harmonic waves in generalized theory of thermoelasticity using three dimensional thermoelasticity with a thermal relaxation time. Subsequently, the Lamb waves in a single lamina where a compact closed-form dispersion relation is derived by separating thermoelastic symmetric and anti-symmetrical modes using trigonometric functions through the lamina thickness.
\end{abstract}

Results: Propagation of guided thermoelastic waves in a homogeneous, orthotropic, thermally conducting plate is investigated within the framework of the generalized theory of thermoelasticity. The results show that both elastic and thermal modes are attenuated, the thermal modes exhibit much larger attenuation than the elastic modes. The attenuation of the former is quite small. Results obtained are extended to a general laminate with an arbitrary layup. On employing boundary conditions on both mid-plane and top surfaces is developed to decouple the wave modes for symmetric and anti-symmetrical modes for laminates.

Conclusions: Propagation of guided thermoelastic waves in a homogeneous, orthotropic, thermally conducting plate is investigated employing generalized theory of thermoelasticity which includes a thermal relaxation time in the heat conduction equation in order to model the finite speed of the thermal wave. Both elastic and thermal modes are attenuated; the thermal modes exhibit much larger attenuation than the elastic modes. The effect of increasing the relaxation time is to lower the speeds of the thermal modes. The effect of anisotropy of the material is quite pronounced on waves propagating in different directions. Thus, it is important to consider the anisotropy of the material in order to accurately model the propagation characteristics.

Keywords: Generalized theory of thermoelasticity; Thermal relaxation time; Composite laminate; Symmetric and antisymmetric modes

Correspondence: klverma@netscape.net

Department of Mathematics, Government Post-Graduate College Hamirpur, Hamirpur 177005, India 


\section{Background}

Composite structures are laminates consisting of several fiber-reinforced laminates that are bonded mutually to attain additional desirable structural properties and enhanced performance than conventional materials. Growing practical applications of advanced composite materials in making structural components, such as aerospace structures, aquatic vehicles, and many other uses has led to the requirement of practical mathematical modeling of their material characteristics and absorption of these models into structural analysis, their strength and stiffness can be tailored to meet stringent intend necessities for high-speed aircrafts, spacecrafts, and space structures. This has resulted in their extensive use in structures that are subjected to severe variations in temperature. In the thermally conducting composite solids, the increases in the temperature distribution due to the occurrence of geometrical or material discontinuities, which may result in the increase of thermal stresses and may often lead to the structural failure of the material. Thermal stresses, especially at the interface between two different materials, often represent a significant aspect in the breakdown of laminated composite structures; as a consequence, a number of mechanical models have been proposed to estimate the importance of thermal loadings (Vel and Batra 2001; Vel and Batra 1999, 2000). Therefore, there is a need to accurately predict thermal stresses in composite structures. For modeling purposes, idealizations are usually made with respect to the geometry and the thermo-mechanical properties of the constituent materials. Accordingly, for fiber-reinforced composites with large values of elastic modulus and thermal conductivity, the fiber can be assumed to be rigid and insulated.

Many investigations (Achenbach 1973; Brekhovskikh 1960; Ewing et al. 1957) have pointed out the highly dispersive character of such waves. There has been a reasonable number of investigations reported in the literature dealing with the analysis of laminated materials, such as Abrate (1998), Choi and Chang (1992), Choi et al. (1991a, b), Chawla (1987), Fukuda et al. (1996), and Halpin (1992), coupled with the development of advanced anisotropic material models of Hayhurst et al. 1999; (Hiermaie et al. 1999). Abo -el-nour and Askar Nadia (2014) determined the bulk acoustic wave (BAW) propagation velocities (quasi-longitudinal, quasi-shear vertical and quasi-shear horizontal) in two important piezoelectric smart materials.

Nayfeh and Chementi (1989) studied dispersion curves of elastic waves in general one-layered anisotropic media, i.e., composite lamina. Nayfeh and Chementi (1991) developed a transfer matrix technique to obtain the dispersion relation curves of elastic wave propagating in multilayered anisotropic media, i.e., composite laminate. However, Nayfeh's formulations in elasticity (Nayfeh 1995;
Nayfeh and Chementi 1989, 1991; Jones 1975, 1999; Liu et al. 1990) and in thermoelasticity (Hawwa and Nayfeh 1995) were limited to a given plane of incidence from a line load parallel to the $y$-axis such that the problem reduces to a generalized plane deformation where all the three displacement components are functions of $x$ and $z$. Verma (2012) studied thermoelastic waves in anisotropic laminated composite plates. Wang and Yuan (2005, 2006) studied damage identification in a composite plate using prestack reverse-time and propagation of lamb wave in composite laminates experimentally. A detailed review can be found in Cementi (1997).

Numerous structural components in space experience a non-uniform temperature variation because of solar radiant heating. Temperature variation through thickness may cause an in-plane expansion or an out-of-plane curvature or both. In order to minimize the thermal deformations in laminated structures composed of layers of material, a precise analytical computation is necessary. In the laminated structures, however, the different material properties of each layer cause large inter-laminar shearing stresses. Consequently, one must also consider thermal stresses in the models of the laminated structures.

The Classical Fourier law of heat conduction and consequent mathematical models for temperature dynamics constructed on the basis of parabolic partial differential equations assumes that the thermal disturbances propagate at infinite speeds. However, the assumption may lead to an inaccurate response of the super large-scale space structures, since a time lag of the propagation of the thermal disturbances in such structures could not be disregarded. The literature dedicated to coupled and generalized theories of thermoelasticity theories is quite large and its detailed review can be found in Nowacki $(1975,1986)$, Chadwick (1960, 1979), and Chadwick and Seet (1970).

The non-classical heat conduction theory that allows the heat disturbances to propagate at finite speeds can be taken into account by using models with thermal relaxation time, which are based on hyperbolic-type equations for temperature. Non-classical heat-conduction theories are closely connected with so-called theories with second sound which view heat propagation as a wave-like phenomenon. The thermal-structural coupling is also considered, since the restriction for the weight of the space structures makes the super large-scale space structures very flexible. Theories, eliminates the paradox of infinite velocity of heat propagation, are called generalized theories of thermoelasticity and its detailed review can be found in Chandrasekharaiah $(1986,1998)$. Theories of Lord and Shulman (1967, referred to as the LS theory) and Green and Lindsay (1972, referred to as the GL theory) are the generalized coupled theory of thermoelasticity. The LS model introduces a single time constant to dictate the relaxation of thermal propagation, as well as the rate of 
change of strain rate and the rate of change of heat generation. In the GL model, on the other hand, the thermal and thermo-mechanical relaxations times are governed by two different time constants.

Achenbach (1973), Banerjee and Pao (1974), and Dhaliwal and Sherief (1980) generalized these theories further to anisotropic heat conducting elastic materials. Hawwa and Nayfeh (1995) studied the general problem of thermoelastic waves in anisotropic periodically laminated composites. Studies of Hawwa and Nayfeh (1996), Thangjitham and Choi (1991), and Tao and Prevost (1984) contain more detailed discussions on this phenomenon. Verma and Hasebe $(2001 ; 2002)$ studied the wave propagation in plates of general anisotropic media in generalized thermoelasticity. Verma et al. (1999) and Verma (2001, 2002) studied thermoelastic problems by considering equation for anisotropic heat-conducting solids with thermal relaxation times.

In this article, a theoretical framework is developed for the displacement and temperature expression of plane harmonic waves using three-dimensional thermoelasticity. Subsequently, the Lamb waves in a single lamina where a compact closed-form dispersion relation is derived by separating thermoelastic symmetric and antisymmetric modes using trigonometric functions through the lamina thickness. A modified exponential form in the thickness direction is projected for deriving the dispersion relation for a composite laminate, with special emphasis on the symmetric laminates.

\section{Methods}

\section{Mathematical formulation}

Consider the Cartesian coordinate system with the direction of $z$-axis normal to the mid-plane of a composite heat-conducting laminate spanned by $x$ - and $y$-axes. Outer surfaces of the laminate are at $z= \pm d / 2$. Thermoelastic Lamb waves propagate in an arbitrary direction $\theta$, which is defined in the counterclockwise relative to the $x$-axis.

Constitutive equations for the linear thermoelastic anisotropic materials in the context of generalized thermoelasticity are stress-strain-temperature relations

$$
\sigma_{i j}=c_{i j k l} \varepsilon_{k l}-\beta_{i j} T
$$

where $\beta_{i j}=c_{i j k l} \alpha_{k l}, \quad i, j, k, l=1,2,3$.

Each layer of the composite laminate with an arbitrary orientation in the global coordinate system $(x, y, z)$ is considered as a monoclinic material having $x-y$ as a plane of symmetry. The stress-strain and temperature relations for monoclinic material therefore take the following matrix form:

$$
\left[\begin{array}{l}
\sigma_{x x} \\
\sigma_{y y} \\
\sigma_{z z} \\
\sigma_{y z} \\
\sigma_{x z} \\
\sigma_{x y}
\end{array}\right]=\left[\begin{array}{cccccc}
c_{11} & c_{12} & c_{13} & 0 & 0 & c_{16} \\
c_{12} & c_{22} & c_{23} & 0 & 0 & c_{26} \\
c_{13} & c_{23} & c_{33} & 0 & 0 & c_{36} \\
0 & 0 & 0 & c_{44} & c_{45} & 0 \\
0 & 0 & 0 & c_{45} & c_{55} & 0 \\
c_{16} & c_{26} & c_{36} & 0 & 0 & c_{66}
\end{array}\right]\left[\begin{array}{c}
\varepsilon_{x x} \\
\varepsilon_{y y} \\
\varepsilon_{z z} \\
\varepsilon_{y z} \\
\varepsilon_{x z} \\
\varepsilon_{x y}
\end{array}\right]-\left[\begin{array}{c}
\beta_{x x} \\
\beta_{y y} \\
\beta_{z z} \\
0 \\
0 \\
\beta_{x y}
\end{array}\right] T
$$

where, since $\sigma_{i j}, \varepsilon_{k l}, \alpha_{k l}$ and $C_{i j k l}$ are tensors, and we are conducting analysis in the global $(x, y, z)$ co-ordinates, any orthogonal transformation of the primed to the non-primed co-ordinates, i.e., $\left(x^{\prime}, y^{\prime}, z^{\prime}\right)$ to $(x, y, z)$ transforms according to (3).

Generally, when the global coordinate system $(x, y, z)$ does not coincide with the principal material coordinate system $\left(x^{\prime}, y^{\prime}, z^{\prime}\right)$ but makes an angle $\phi$ with the $z$-axis, the stiffness matrix $c_{i j k l}\left(c_{i j}\right)$ system can be obtained from the stiffness matrix $c_{i j k l}^{\prime}\left(c_{i j}^{\prime}\right)$ system by using transformations

$$
\begin{aligned}
c_{11}= & c_{11}^{\prime} u^{4}+c_{22}^{\prime} v^{4}+2\left(c_{12}^{\prime}+2 c_{66}^{\prime}\right) u^{2} v^{2}, \\
c_{12}= & \left(c_{11}^{\prime}+c_{22}^{\prime}-4 c_{66}^{\prime}\right) u^{2} v^{2}+c_{12}^{\prime}\left(u^{4}+v^{4}\right), \\
c_{13}= & c_{13}^{\prime} u^{2}+c_{23}^{\prime} v^{2}, c_{16}=\left(c_{11}^{\prime}-c_{12}^{\prime}-2 c_{66}^{\prime}\right) u^{3} v \\
& +\left(c_{12}^{\prime}-c_{22}^{\prime}+2 c_{66}^{\prime}\right) v^{3} u, \\
c_{22}= & c_{11}^{\prime} v^{4}+c_{22}^{\prime} u^{4}+2\left(c_{12}^{\prime}+2 c_{66}^{\prime}\right) u^{2} v^{2}, \\
c_{23}= & c_{23}^{\prime} u^{2}+c_{13}^{\prime} v^{2}, \\
c_{26}= & \left(c_{11}^{\prime}-c_{12}^{\prime}-2 c_{66}^{\prime}\right) u v^{3}+\left(c_{12}^{\prime}-c_{22}^{\prime}+2 c_{66}^{\prime}\right) v u^{3}, \\
c_{33}= & c_{33}^{\prime}, c_{36}=\left(c_{23}^{\prime}-c_{13}^{\prime}\right) u v, c_{45}=\left(c_{44}^{\prime}-c_{55}^{\prime}\right) u v, \\
c_{44}= & c_{44}^{\prime} u^{2}+c_{55}^{\prime} v^{2}, c_{55}=c_{55^{\prime}} u^{2}+c_{44}^{\prime} v^{2}, \\
c_{66}= & \left(c_{11}^{\prime}+c_{22}^{\prime}-2 c_{12}^{\prime}-2 c_{66}^{\prime}\right) u^{2} v^{2}+c_{16}^{\prime}\left(u^{4}+v^{4}\right), \\
\alpha_{12}= & \left(\alpha_{33}^{\prime}-\alpha_{11}^{\prime}\right) u v, \beta_{x x}=\beta_{x x}^{\prime} u^{2}+\beta_{z z}^{\prime} v^{2}, \\
\beta_{y y}= & \beta_{x x}^{\prime} v^{2}+\beta_{z z}^{\prime} u^{2}, \beta_{x y}=\left(\beta_{z z^{\prime}}^{\prime}-\beta_{x x}^{\prime}\right) u v, \beta_{z z}=\beta_{z z}^{\prime}
\end{aligned}
$$

where $u=\cos \varphi$ and $v=\sin \varphi$.

The linear strain-displacement relations are

$$
\begin{aligned}
\varepsilon_{x y} & =\frac{1}{2}\left(\frac{\partial u}{\partial y}+\frac{\partial v}{\partial x}\right), \varepsilon_{y z}=\frac{1}{2}\left(\frac{\partial v}{\partial z}+\frac{\partial w}{\partial y}\right) \\
\varepsilon_{z x} & =\frac{1}{2}\left(\frac{\partial u}{\partial z}+\frac{\partial w}{\partial x}\right), \varepsilon_{x x}=\frac{\partial u}{\partial x} \\
\varepsilon_{y y} & =\frac{\partial v}{\partial y}, \varepsilon_{z z}=\frac{\partial w}{\partial z}
\end{aligned}
$$

The summation convention is implied; $u, v$ and $w$ are the displacements in the $x, y$, and $z$ directions and $\sigma_{i j}$ and $\varepsilon_{i j}$ are the stress and strain tensors, respectively; $\beta_{i j}$ is the thermal modulus; $\alpha_{i j}$ is the thermal expansion tensor; $T$ is the temperature; and the fourth-order tensor of the elasticity $C_{i j k l}$ satisfies the (Green) symmetry conditions:

$$
c_{i j k l}=c_{k l i j}=c_{i j l k}=c_{j i k l}, \varepsilon_{i j}=\varepsilon_{j i} \text { and } \alpha_{i j}=\alpha_{j i}, \beta_{i j}=\beta_{j i}, K_{i j}=K_{j i}
$$


The equations of motion and energy are given by

$$
\begin{aligned}
& {\left[c_{i j k l} \frac{\partial^{2} u_{k}}{\partial x_{j} \partial x_{l}}-\beta_{i j} \frac{\partial T}{\partial x_{j}}\right]=\rho \frac{\partial^{2} u_{i}}{\partial t^{2}}} \\
& K_{i j} \frac{\partial^{2} T}{\partial x_{i} \partial x_{j}}-\rho C_{e}\left(\frac{\partial T}{\partial t}+\tau_{0} \frac{\partial^{2} T}{\partial^{2} t}\right)=T_{0} \beta_{i j}\left(\frac{\partial}{\partial t}+\tau_{0} \frac{\partial^{2}}{\partial^{2} t}\right) \frac{\partial u_{i}}{\partial x_{j}}
\end{aligned}
$$

where $\rho$ is the density, $t$ is the time, $K_{i j}$ are the thermal conductivities, $C_{e}$ and $\tau_{0}$ are respectively the specific heat at constant strain, and thermal relaxation time.

\section{Analysis}

For transient waves propagating in the $x-y$ plane of the plate in a direction of angle $\phi$ with respect to the $x$ axis, the displacements have the form

$$
\begin{aligned}
\left(u_{1}, u_{2}, u_{3}, T\right)= & (U(z), V(z), W(z), \Theta(z)) e^{i\left[\left(\ell_{x} x+\ell_{y} y\right)-\omega t\right]} \\
& \times i=\sqrt{-1}
\end{aligned}
$$

where $\mathbf{k}=\left[\ell_{x}, \ell_{y}\right]^{T}$ is the wave vector, and its magnitude,

$$
|\mathbf{k}|=k=\sqrt{\ell^{2}{ }_{x}+\ell^{2}}={ }^{\omega} / c_{p},
$$

is the wave number, $\omega$ is the angular frequency, and $c_{p}$ is the phase velocity. Here, $\mathbf{k}$ points in the direction of propagation. Substituting Equation 7 into Equations 2 via 4, we obtain

$$
\begin{aligned}
\sigma_{x x}= & {\left[c_{11} \ell_{x} U+c_{12} \ell_{y} V-i c_{13} W^{\prime}+c_{16}\left(\ell_{y} U+\ell_{x} V\right)+i \beta_{x x} T\right] E } \\
\sigma_{y y}= & {\left[c_{12} \ell_{x} U+c_{22} \ell_{y} V-i c_{23} W^{\prime}+c_{26}\left(\ell_{y} U+\ell_{x} V\right)+i \ell_{y} T\right] E } \\
\sigma_{z z}= & {\left[c_{13} \ell_{x} U+c_{23} \ell_{y} V-i c_{33} W^{\prime}+c_{36}\left(\ell_{y} U+\ell_{x} V\right)+i \beta_{z} T\right] E } \\
\sigma_{y z}= & {\left[c_{44}\left(V^{\prime}+i \ell_{y} W\right)+c_{45}\left(U^{\prime}+i \ell_{x} W\right)\right] E } \\
\sigma_{x z}= & {\left[c_{45}\left(V^{\prime}+i \ell_{y} W\right)+c_{55}\left(U^{\prime}+i \ell_{x} W\right)\right] E } \\
\sigma_{x y}= & {\left[c_{16} \ell_{x} U+c_{26} \ell_{y} V-i c_{36} W^{\prime}\right.} \\
& \left.+c_{66}\left(\ell_{y} U+\ell_{x} V\right)+i \beta_{x y} T\right] E
\end{aligned}
$$

where $E=i e^{i\left[\left(\ell_{x} x+\ell_{y} y\right)-\omega t\right]}$

The thermal gradient

$$
\frac{\partial T}{\partial z}=\Theta^{\prime}(z) e^{i\left[\left(\ell_{x} x+\ell_{y} y\right)-\omega t\right]}
$$

where the prime indicates the derivative with respect to $z$. Substituting Equations 9 into the equation of motion and heat conduction Equations 5 and 6 for monoclinic material, gives

$$
\begin{aligned}
& -c_{55} U^{\prime \prime}-c_{45} V^{\prime \prime}+\left(c_{11} \ell_{x}^{2}+2 c_{16} \ell_{x} \ell_{y}+c_{66} \ell_{y}^{2}-\rho \omega^{2}\right) U \\
& +\left[c_{16} \ell_{x}^{2}+\left(c_{12}+c_{66}\right) \ell_{x} \ell_{y}+c_{26} \ell_{y}^{2}\right] V-i\left[\left(c_{13}+c_{55}\right) \ell_{x}\right. \\
& \left.\left.+\left(c_{36}+c_{45}\right) \ell_{y}\right)\right] W^{\prime}-i\left[\beta_{x} \ell_{x}+\beta_{x y} \ell_{y}\right] \Theta=0
\end{aligned}
$$

$$
\begin{aligned}
& -c_{45} U^{\prime \prime}-c_{44} V^{\prime \prime}+\left[c_{16} \ell_{x}^{2}+\left(c_{12}+c_{66}\right) \ell_{x} \ell_{y}+c_{26} \ell_{y}^{2}\right] U \\
& +\left(c_{66} \ell_{x}^{2}+2 c_{26} \ell_{x} \ell_{y}+c_{22} \ell_{y}^{2}-\rho \omega^{2}\right) V \\
& -i\left[\left(c_{36}+c_{45}\right) \ell_{x}+\left(c_{23}+c_{44}\right) \ell_{y}\right] W^{\prime} \\
& -i\left(\beta_{x y} \ell_{x}+\beta_{y} \ell_{y}\right] \Theta=0
\end{aligned}
$$

$$
\begin{aligned}
& -i\left[\left(c_{13}+c_{55}\right) \ell_{x}+\left(c_{36}+c_{45}\right) \ell_{y}\right] U^{\prime} \\
& -i\left[\left(c_{36}+c_{45}\right) \ell_{x}+\left(c_{23}+c_{44}\right) \ell_{y}\right] V^{\prime}-c_{33} W^{\prime \prime} \\
& +\left[c_{55} \ell_{x}^{2}+2 c_{45} \ell_{x} \ell_{y}+c_{44} \ell_{y}^{2}-\rho \omega^{2}\right] W-\beta_{z} \Theta^{\prime}=0 \\
& -k_{33} \Theta^{\prime \prime}+\left[k_{11} \ell_{x}^{2}+2 k_{12} \ell_{x} \ell_{y}+c_{22} \ell_{y}^{2}-\rho c_{p}^{2} C_{e} \tau\right) \Theta \\
& -T_{0} i k c_{p}^{2} \tau\left\{\left(\beta_{x} \ell_{x}+\beta_{x y} \ell_{y}\right) U\right. \\
& -\left(\beta_{x y} \ell_{x}+\beta_{y} \ell_{y}\right) V+i \beta_{z} W^{\prime}=0
\end{aligned}
$$

\section{Waves in a composite lamina}

In an off-axis lamina, the solutions of Equations 10 can be simply separated into symmetric and antisymmetric wave modes, which make the analytical representation particularly simple:

$$
\begin{aligned}
& \left(U_{s}, V_{s}, W_{s}, \Theta_{s}\right)=\left(A_{s} \cos \alpha z, B_{s} \cos \alpha z, C_{s} \sin \alpha z, D_{s} \cos \alpha z\right) \\
& \left(U_{a}, V_{a}, W_{a}, \Theta_{a}\right)=\left(A_{a} \sin \alpha z, B_{a} \sin \alpha z, C_{a} \cos \alpha z, D_{a} \sin \alpha z\right)
\end{aligned}
$$

Substituting these expressions into the equation of motion and heat conduction equation is an unknown variable $\alpha$ to be determined later; moreover, the subscripts $s$ and $a$ represent symmetric and antisymmetric modes, respectively. First, substituting the symmetric mode into the equations of motion, Equation 10 may be expressed in a matrix form

$$
\left[\begin{array}{cccc}
\Gamma_{11}-\rho c_{p}^{2} & \Gamma_{12} & \Gamma_{13} & \Gamma_{14} \\
\Gamma_{12} & \Gamma_{22}-\rho c_{p}^{2} & \Gamma_{23} & \Gamma_{24} \\
\bar{\Gamma}_{13} & \bar{\Gamma}_{23} & \Gamma_{33}-\rho c_{p}^{2} & \Gamma_{34} \\
\bar{\Gamma}_{41} & \bar{\Gamma}_{42} & \Gamma_{43} & \Gamma_{44}-\frac{\rho \omega^{2} C_{e} T_{0} \tau}{T_{0} \omega^{2} \tau}
\end{array}\right]\left[\begin{array}{c}
A_{s} \\
B_{s} \\
C_{s} \\
D_{s}
\end{array}\right]=0
$$

where the bar indicates complex conjugate. The elements in the above matrix defined by $\left(\boldsymbol{\Gamma}-\rho \omega^{2} \mathbf{I}\right)$ are as follows: 


$$
\begin{aligned}
\Gamma_{11}= & c_{11} \ell_{x}^{2}+2 c_{16} \ell_{x} \ell_{y}+c_{66} \ell_{y}^{2}+c_{55} \alpha^{2}, \\
\Gamma_{12}= & c_{16} \ell_{x}^{2}+\left(c_{12}+c_{66}\right) \ell_{x} \ell_{y}+c_{26} \ell_{y}^{2}+c_{45} \alpha^{2}, \\
\Gamma_{14}= & -i\left[\beta_{x x} \ell_{x}+\beta_{x y} \ell_{y}\right], \\
\Gamma_{22}= & c_{66} \ell_{x}^{2}+2 c_{26} \ell_{x} \ell_{y}+c_{22} \ell_{y}^{2}+c_{44} \alpha^{2}, \\
\Gamma_{23}= & -i\left[\left(c_{36}+c_{45}\right) l_{x}+\left(c_{23}+c_{44}\right) l_{y}\right] \alpha, \\
\Gamma_{24}= & -i\left[\beta_{x y} \ell_{x}+\beta_{y y} \ell_{y}\right], \\
\Gamma_{31}= & i\left[\left(c_{13}+c_{55}\right) \ell_{x}+\left(c_{36}+c_{45}\right) \ell_{y}\right] \alpha=\bar{\Gamma}_{13}, \\
\Gamma_{32}= & \left.i\left(c_{36}+c_{45}\right) \ell_{x}+\left(c_{23}+c_{44}\right) \ell_{y}\right] \alpha=\bar{\Gamma}_{23}, \\
\Gamma_{33}= & c_{55} \ell_{x}^{2}+2 c_{45} \ell_{x} \ell_{y}+c_{44} \ell_{y}^{2}+c_{33} \alpha^{2}, \\
\Gamma_{34}= & \beta_{z} \alpha \\
\Gamma_{41}= & i\left(\beta_{x} \ell_{x}+\beta_{x y} \ell_{y}\right)=\bar{\Gamma}_{14}, \Gamma_{41}=i\left(\beta_{x x} \ell_{x}+\beta_{x y} \ell_{y}\right)=\bar{\Gamma}_{14}, \\
\Gamma_{42}= & i\left(\beta_{x y} \ell_{x}+\beta_{y y} \ell_{y}\right)=\bar{\Gamma}_{24}, \\
\Gamma_{43}= & \beta_{z z} \alpha=\Gamma_{34}, \\
\Gamma_{44}= & -\left[k_{11} \ell_{x}^{2}+2 k_{12} \ell_{x} \ell_{y}+k_{22} \ell_{y}^{2}+k_{33} \alpha^{2}\right] / T_{0} \omega^{2} \tau, \\
& \tau=\tau_{0}+{ }^{i} / \omega
\end{aligned}
$$

For nontrivial solutions of $A_{s}, B_{s}, C_{s}$ and $D_{s}$ in Equation 12, the determinant of the $4 \times 4$ matrix vanishes, which leads to the following eight-degree polynomial in terms of $\alpha$ :

$$
\begin{aligned}
& P_{0} \alpha^{8}+\left(P_{1}+\varepsilon C_{0}\right) \alpha^{6}+\left(P_{2}+\varepsilon C_{1}\right) \alpha^{4} \\
& +\left(P_{3}+\varepsilon C_{2}\right) \alpha^{2}+\left(P_{4}+\varepsilon C_{3}\right)=0
\end{aligned}
$$

Since Equation 14 is a biquadratic equation in $\alpha^{2}$, it has four roots for $\alpha^{2}\left(\alpha_{j}, j=1,2,3,4\right)$.

Hence, the eight roots for $\alpha$ can be arranged in four pairs as

$$
\alpha_{j+1}=-\alpha_{j}, \quad(j=1,3,5,7)
$$

In Equation 14, $\varepsilon$ is a coupling constant, if coupling constant is zero, then Equation 14 reduces to

$$
\begin{aligned}
P_{0} \alpha^{8}+ & P_{1} \alpha^{6}+P_{2} \alpha^{4}+P_{3} \alpha^{2}+P_{4}=0, \\
P_{0}= & \left(c_{45}^{2} c_{33}-c_{33} c_{44} c_{55}\right) k_{33} \\
P_{1}= & \left(c_{33} c_{44} c_{55}-c_{45}^{2} c_{33}\right) F_{4} \\
& +\left\{\begin{array}{l}
\left(c_{45}^{2}-c_{44} c_{55}\right) F_{3}-c_{33} c_{55} F_{2}-c_{33} c_{44} F_{1} \\
+\left(c_{55} F_{23}-c_{45} F_{13}\right) F_{32}+\left(c_{44} F_{13}-c_{45} F_{23}\right) F_{31} \\
+2 c_{33} c_{45} F_{12}
\end{array}\right\} k_{33} \\
P_{2}=\left\{\begin{array}{l}
\left(c_{44} c_{55}-c_{45}^{2}\right) F_{3}+c_{33} c_{55} F_{2}+c_{33} c_{44} F_{1}-2 c_{33} c_{45} F_{12} \\
-\left(c_{44} F_{13}-c_{45} F_{23}\right) F_{31}-\left(c_{55} F_{23}-c_{45} F_{13}\right) F_{32}
\end{array}\right\} F_{4} & \\
& +\left\{\begin{array}{l}
\left(F_{32} F_{23}-c_{44} F_{3}-c_{33} F_{2}\right) F_{1}+\left(F_{31} F_{13}-c_{55} F_{3}\right) F_{2} \\
+2 c_{45} F_{12} F_{3}-\left(F_{13} F_{32}+F_{23} F_{31}\right) F_{12}+c_{33} F_{12}^{2}
\end{array}\right\} k_{33} \\
P_{3}= & \left\{\begin{array}{l}
\left(c_{55} F_{2}+c_{44} F_{1}-2 c_{45} F_{12}\right) F_{3}+\left(c_{33} F_{1}-F_{31} F_{13}\right) F_{2} \\
+\left(F_{13} F_{12}-F_{1} F_{23}\right) F_{32}+F_{12} F_{23} F_{31}-c_{33} F_{12}^{2}
\end{array}\right\} F_{4} \\
& -\left(F_{1} F_{2}-F_{12}^{2}\right) F_{3} k_{33} \\
P_{4}= & \left(F_{1} F_{2}-F_{12}^{2}\right) F_{3} F_{4} \\
C_{0}= & \left(c_{45}^{2}-c_{44} c_{55}\right) F_{34} F_{43}
\end{aligned}
$$

$$
\begin{aligned}
& C_{1}=\left(c_{33} c_{55} F_{42}-c_{33} c_{45} F_{41}+c_{45} F_{31} F_{43}-c_{55} F_{32} F_{43}\right)\left(-F_{24}\right) \\
& +\left(2 c_{45} F_{12}-c_{55} F_{2}-c_{44} F_{1}\right) F_{34} F_{43} \\
& +\left(c_{44} F_{14} F_{31}-c_{45} F_{14} F_{32}\right) F_{43} \\
& +\left(c_{44} F_{13}-c_{45} F_{23}\right) F_{34} F_{41}-c_{33} c_{44} F_{14} F_{41} \\
& +\left(c_{55} F_{23}-c_{45} F_{13}\right) F_{34} F_{42}+c_{33} c_{45} F_{14} F_{42} \\
& C_{2}=\left(\begin{array}{l}
\left(c_{33} F_{42}-F_{43} F_{32}\right) F_{1}+\left(c_{55} F_{42}-c_{45} F_{41}\right) F_{3} \\
+\left(F_{13} F_{32}-c_{33} F_{12}\right) F_{41}+\left(F_{12} F_{43}-F_{13} F_{42}\right) F_{31}
\end{array}\right)\left(-F_{24}\right) \\
& +\left(F_{23} F_{42}-F_{2} F_{43}\right) F_{34} F_{1} \\
& +\left(F_{31} F_{14} F_{43}+F_{13} F_{41} F_{34}-C_{33} F_{14} F_{41}\right) F_{2} \\
& +\left(F_{23} F_{32}-c_{44} F_{3}\right) F_{14} F_{41} \\
& +\left(F_{12} F_{34}-F_{14} F_{32}\right) F_{43} F_{12} \\
& -\left(F_{13} F_{42}+F_{23} F_{41}\right) F_{12} F_{34} \\
& +\left(c_{45} F_{3}+c_{33} F_{12}-F_{31} F_{23}\right) F_{14} F_{42} \\
& C_{3}=F_{3}\left(F_{41} F_{12}-F_{1} F_{42}\right) F_{24}-F_{14} F_{3}\left(F_{41} F_{2}-F_{42} F_{12}\right) \\
& F_{12}=c_{16} \ell_{x}^{2}+\left(c_{12}+c_{66}\right) \ell_{x} \ell_{y}+c_{26} \ell_{y}^{2}, \\
& F_{13}=-i\left(\left(c_{13}+c_{55}\right) \ell_{x}+\left(c_{36}+c_{45}\right) \ell_{y}\right) \\
& F_{14}=-i\left(\beta_{x x} \ell_{x}+\beta_{x y} \ell_{y}\right), \\
& F_{24}=-i\left(\beta_{x y} \ell_{x}+\beta_{y y} \ell_{y}\right) \\
& F_{23}=-i\left(\left(c_{36}+c_{45}\right) \ell_{x}+\left(c_{23}+c_{44}\right) \ell_{y}\right) \\
& F_{1}=c_{11} \ell_{x}^{2}+2 c_{16} \ell_{x} \ell_{y}+c_{66} \ell_{y}^{2}, \\
& F_{2}=c_{66} \ell_{x}^{2}+2 c_{26} \ell_{x} \ell_{y}+c_{22} \ell_{y}^{2} \\
& F_{3}=c_{55} \ell_{x}^{2}+2 c_{45} c_{16} \ell_{x} \ell_{y}+c_{44} \ell_{y}^{2} \text {, } \\
& F_{4}=-\left(K_{11} \ell_{x}^{2}+2 K_{12} \ell_{x} \ell_{y}+K_{22} \ell_{y}^{2}\right) \\
& F_{21}=c_{16} \ell_{x}^{2}+\left(c_{12}+c_{66}\right) \ell_{x} \ell_{y}+c_{26} \ell_{y}^{2}=F_{12}, \\
& F_{31}=i\left(\left(c_{13}+c_{55}\right) \ell_{x}+\left(c_{36}+c_{45}\right) \ell_{y}\right) \\
& F_{32}=i\left(\left(c_{36}+c_{45}\right) \ell_{x}+\left(c_{23}+c_{44}\right) \ell_{y}\right) \text {, } \\
& F_{11}=i\left(\beta_{x x} \ell_{x}+\beta_{x y} \ell_{y}\right) \text {, } \\
& F_{42}=i\left(\beta_{x y} \ell_{x}+\beta_{y y} \ell_{y}\right), F_{34}=F_{43}=\beta_{z z} \text {. }
\end{aligned}
$$

Here $\varepsilon$ is a coupling parameter, and for each $\xi_{j}$, in symmetric modes, $A_{s}, B_{s}, C_{s}$, and $D_{s}$ can be expressed in terms of $A_{s}$ via Equation 12 as

$$
\begin{aligned}
B_{s} & =\Delta_{1} / \Delta=R A_{s}, C_{s}=\Delta_{2} / \Delta=i S A_{s}, D_{s} \\
& =\Delta_{3} / \Delta=\Omega A_{s}
\end{aligned}
$$

Where

$$
\begin{aligned}
\Delta= & \left(c_{33} c_{44} F_{14}-c_{33} c_{45} F_{24}+\left(c_{45} F_{23}-c_{44} F_{13}\right) F_{34}\right) \alpha^{4} \\
& +\left(\left(c_{33} F_{2}+c_{44} F_{3}-F_{23} F_{32}\right) F_{14}+\left(F_{12} F_{23}-F_{2} F_{13}\right) F_{34}\right. \\
& \left.+\left(F_{13} F_{32}-c_{45} F_{3}-c_{33} F_{12}\right) F_{24}\right) \alpha^{2}+\left(F_{14} F_{2}-F_{24} F_{12}\right) F_{3} \\
& \\
\Delta_{1}= & \left(c_{33} c_{55} F_{24}-c_{33} c_{45} F_{14}+\left(c_{45} F_{13}-c_{55} F_{23}\right) F_{34}\right) \alpha^{4} \\
& +\left(\left(c_{33} F_{1}+c_{55} F_{3}-F_{13} F_{31}\right) F_{24}+\left(F_{12} F_{13}-F_{1} F_{23}\right) F_{34}\right. \\
& \left.+\left(F_{13} F_{23}-c_{45} F_{3}-c_{33} F_{12}\right) F_{14}\right) \alpha^{2}+\left(F_{24} F_{1}-F_{14} F_{12}\right) F_{3}
\end{aligned}
$$




$$
\begin{aligned}
\Delta_{2}= & \alpha\left\{\begin{array}{l}
\left(c_{44} c_{55}-c_{45}^{2}\right) F_{34} \alpha^{4} \\
+\left(\begin{array}{l}
\left(c_{45} F_{14}-c_{55} F_{24}\right) F_{32}+\left(c_{45} F_{24}-c_{44} F_{14}\right) F_{31} \\
+\left(c_{44} F_{1}+c_{55} F_{2}-2 c_{45} F_{12}\right) F_{34}
\end{array}\right) \alpha^{2}
\end{array}\right\} \\
& +\left(F_{12} F_{24}-F_{2} F_{14}\right) F_{31}+\left(F_{12} F_{14}-F_{1} F_{24}\right) F_{32} \\
& +\left(F_{1} F_{2}-F_{12}^{2}\right) F_{34} \\
\Delta_{3}= & \left(c_{45}^{2} c_{33}-c_{33} c_{44} c_{55}\right) \alpha^{6} \\
& +\left(\begin{array}{l}
\left(c_{45}^{2}-c_{44} c_{55}\right) F_{3}+\left(c_{44} F_{13}-c_{45} F_{23}\right) F_{31} \\
+\left(c_{55} F_{23}-c_{45} F_{13}\right) F_{32}+c_{33}\left(2 F_{12} c_{45}-c_{55} F_{2}-c_{44} F_{1}\right)
\end{array}\right) \alpha^{4} \\
& +\left(\left(c_{33} F_{12}-F_{23} F_{31}+2 c_{45} F_{3}-F_{13} F_{32} F_{12}+F_{2} F_{13} F_{31}\right.\right. \\
& \left.+F_{1} F_{23} F_{32}-c_{33} F_{1} F_{2}-c_{55} F_{2} F_{3}-c_{44} F_{1} F_{3}\right) \alpha^{2}+F_{12}^{2} F_{3}-F_{1} F_{2} F_{3}
\end{aligned}
$$

and similarly for antisymmetric modes, $B_{a}=R A_{a}$, $C_{a}=-i S A_{a}$ and $D_{a}=\Omega A_{a}$. With the above equations, the polarization displacement vectors and temperature are determined from the three roots. Consequently, the general solution of Equation 11 is

$$
\begin{aligned}
& \left(U_{s}, V_{s}, W_{s}, \Theta_{s}\right)=\sum_{j=1}^{4} A_{s j}\left\{\begin{array}{l}
\cos \alpha_{j} z, R_{j} \cos \alpha_{j} z \\
\left.i S_{j} \sin \alpha_{j} z, \Omega_{j} \cos \alpha_{j} z\right\}
\end{array}\right. \\
& \left(U_{a}, V_{a}, W_{a}, \Theta_{a}\right)=\sum_{j=1}^{4} A_{a j}\left\{\begin{array}{l}
\sin \alpha_{j} z, R_{j} \sin \alpha_{j} z \\
\left.-i S_{j} \cos \alpha_{j} z, \Omega_{j} \sin \alpha_{j} z\right\}
\end{array}\right.
\end{aligned}
$$

Substituting Equation 17 into Equation 9, the expression of $\sigma_{z z}, \sigma_{y z}, \sigma_{x z}$, and $\frac{\partial T}{\partial z}$ with traction and thermal gradient-free boundary conditions on the top and bottom surfaces $z= \pm^{h} / 2$, then $\sigma_{z z}, \sigma_{y z}, \sigma_{x z}$, and $\frac{\partial T}{\partial z}$ may be expressed for the symmetric and antisymmetric modes respectively as

$$
\left.\left(\sigma_{z z}, \sigma_{y z}, \sigma_{x z}, \frac{\partial T}{\partial z}\right)\right|_{z=h / 2}=\sum_{j=1}^{4}\left\{\begin{array}{l}
N_{1 j}\left(\begin{array}{c}
\cos \alpha_{j} z \\
\sin \alpha_{z} z
\end{array}\right), N_{2 j}\left(\begin{array}{c}
\sin \alpha_{j} z \\
\cos \alpha_{j} z
\end{array}\right), A_{3 j}\left(\begin{array}{c}
\sin \alpha_{j} z \\
\cos \alpha_{j} z
\end{array}\right), N_{4 j}\left(\begin{array}{c}
\sin \alpha_{j} z \\
\cos \alpha_{j} z
\end{array}\right)
\end{array}\right\} A_{j}
$$

$$
\begin{aligned}
\mathrm{N}_{1 j}= & c_{13} \ell_{x}+c_{23} \ell_{y} R_{j}+c_{33} \alpha_{j} S_{j}+c_{36}\left(\ell_{y}+\ell_{x} R_{j}\right) \\
& +i \beta_{z} \Omega_{j} \\
\mathrm{~N}_{2 j}= & c_{44}\left(\alpha_{j} R_{j}+\ell_{y} S_{j}\right)+c_{45}\left(\alpha_{j}+i \ell_{x} S_{j}\right) \\
\mathrm{N}_{3 j}= & c_{45}\left(\ell_{j} R_{j}+\alpha_{y} S_{j}\right)+c_{55}\left(\alpha_{j}+\ell_{x} S_{j}\right) \\
\mathrm{N}_{4 j}= & -\alpha_{j} \Omega_{j}
\end{aligned}
$$

The existence of a nontrivial solution of Equation 18 leads to closed-form dispersion relations as

$$
\begin{aligned}
& N_{11} G_{1} \tan \left(\alpha_{1} h / 2+\chi\right)+N_{12} G_{3} \tan \left(\alpha_{2} h / 2+\chi\right) \\
& \quad+N_{13} G_{5} \tan \left(\alpha_{3} h / 2+\chi\right)+N_{14} G_{7} \tan \left(\alpha_{4} h / 2+\chi\right)=0
\end{aligned}
$$

$$
\begin{aligned}
G_{1} & =\left|\begin{array}{lll}
N_{22} & N_{23} & N_{24} \\
N_{32} & N_{33} & N_{34} \\
N_{42} & N_{43} & N_{44}
\end{array}\right|, \\
G_{2} & =\left|\begin{array}{lll}
N_{21} & N_{23} & N_{24} \\
N_{31} & N_{33} & N_{34} \\
N_{41} & N_{43} & N_{44}
\end{array}\right|, \\
G_{3} & =\left|\begin{array}{lll}
N_{21} & N_{22} & N_{24} \\
N_{31} & N_{32} & N_{34} \\
N_{41} & N_{42} & N_{44}
\end{array}\right|, \\
G_{4} & =\left|\begin{array}{lll}
N_{21} & N_{22} & N_{23} \\
N_{31} & N_{32} & N_{33} \\
N_{41} & N_{42} & N_{43}
\end{array}\right|
\end{aligned}
$$

where $X=0$ and $X=\pi / 2$ represent antisymmetric and symmetric Lamb wave modes, respectively. Equation 20 is a transcendental equation implicitly relating $\omega$ to $\mathbf{k}$. For a fixed $\theta$, a numerical iterative root-finding method is employed to compute the admissible $\omega$ for a range of $\mathbf{k}$ values, leading to dispersion relations of Lamb wave modes in the direction of propagation. Furthermore, in general, the frequency $\omega$ of each mode is the single-valued function of $\mathbf{k}$.

\section{Waves in a composite laminate}

In formulating thermoelastic Lamb waves in a heatconducting laminate, the interfaces between layers are assumed to be perfectly bonded. The displacement and temperature components of each layer in the $z$ axis Equation 11 needs to be modified in exponential forms to accommodate the inhomogeneity of the multilayered laminates.

$$
\begin{aligned}
& U=A e^{i \alpha z}, V=B e^{i \alpha z}, W=-i C e^{i \alpha z} \\
& \Theta_{s}=D e^{i \alpha z}
\end{aligned}
$$

Substituting these expressions into the equations of motion, Equation 10 may be rearranged in a matrix form.

For nontrivial solutions of $A, B, C$ and $D$ in Equation 12, the determinant of the $4 \times 4$ matrix vanishes,

$$
\left[\begin{array}{cccc}
\Gamma_{11}-\rho c_{p}^{2} & \Gamma_{12} & \Gamma_{13} & \Gamma_{14} \\
\Gamma_{12} & \Gamma_{22}-\rho c_{p}^{2} & \Gamma_{23} & \Gamma_{24} \\
\bar{\Gamma}_{13} & \bar{\Gamma}_{23} & \Gamma_{33}-\rho c_{p}^{2} & \Gamma_{34} \\
\bar{\Gamma}_{41} & \bar{\Gamma}_{42} & \Gamma_{43} & \Gamma_{44}-\frac{\rho \omega^{2} C_{e} T_{0} \tau}{T_{0} \omega^{2} \tau}
\end{array}\right]\left[\begin{array}{c}
A \\
B \\
C \\
D
\end{array}\right]=0
$$

giving the following eight-degree polynomial in terms of $\alpha$, which is a biquadratic equation, it has four roots for $\alpha^{2}\left(\alpha_{j}, j=1,2,3,4\right)$, and these eight roots for $\alpha$ can be arranged in four pairs as

$$
\alpha_{j+1}=-\alpha_{j}, \quad(j=1,3,5,7)
$$


For each $\alpha_{j}, B, C$ and $D$ can be expressed in terms of $A$ via Equation 23 as $B=R A, C=-S A$ and $D=\Omega A$.

Thus, the general solution of Equations 22 and 23 in each lamina is

$$
(U, V, W, T)=E \sum_{j=1}^{8}\left(A_{j}, R_{j} A_{j}, S_{j} A_{j}, \Omega_{j} A_{j}\right) e^{i k \alpha_{j} z}
$$

The inter-laminar stress and thermal gradient components, $\sigma_{z z}, \sigma_{y z}, \sigma_{x z}$ and $\frac{\partial T}{\partial z}$ in each lamina may be expressed as

$$
\begin{aligned}
\left.\left(\sigma_{z z}, \sigma_{y z}, \sigma_{x z}, \frac{\partial T}{\partial \mathrm{z}}\right)\right|_{z= \pm h / 2}= & i k e^{i\left[\left(\left[\left(\ell_{x} x+\ell_{y} y\right)-\omega t\right]\right.\right.} \sum_{j=1}^{8} \\
& \left(N_{1 j}, N_{2 j}, N_{3 j}, N_{4 j}\right) A_{j} e^{i k \alpha_{j} z}
\end{aligned}
$$

Generally, there are two methods, namely transfer matrix method and assemble matrix method, for obtaining the dispersion relations in laminates. Although the procedures of these two methods seem different, they are identical in principle by both satisfying traction-free boundary conditions on the outer surfaces of the laminate and continuity of interface conditions between two adjacent laminas in a different manner. Both methods can calculate dispersion curves in a general laminate with an arbitrary stacking sequence. Using Equations 22 and 23, it may be observed that symmetric and antisymmetric wave modes in general laminates cannot be decoupled. However, in designing the composite structures, symmetric laminates are practically used. A robust method is proposed to separate the two types of wave modes by imposing boundary conditions at both top and mid-plane surface. Traction-free boundary conditions on the top surface of the laminate are given by

$$
\left.\left(\sigma_{z z}, \sigma_{y z}, \sigma_{x z}, \frac{\partial T}{\partial \mathrm{z}}\right)\right|_{z=h / 2}=0
$$

Because of the symmetric geometry and symmetric material property of the laminate, only half of the laminate needs to be considered and then the following conditions on the stress and displacement components at the midplane for symmetric modes are imposed

$$
\left.\left(w, \sigma_{y z}, \sigma_{x z}, \frac{\partial T}{\partial z}\right)\right|_{z=0}=0
$$

Likewise, the boundary conditions of antisymmetric modes at the mid-plane are

$$
\left.\left(u, v, \sigma_{z z}, T\right)\right|_{z=0}=0
$$

By imposing displacement and stress continuity conditions along the interfaces of half lay-up of an $N$-layered laminate, a total of $4 N$ equations are constructed if the assemble matrix method is used. Then set the determinant of the $4 N$ equations to zero, and numerically solve the resulting transcendental equation for the dispersion relations of Lamb waves in symmetric laminates.

\section{Results and discussion}

The formulation and analysis described in the preceding sections has been implemented, because it can seamlessly combine symbolic and numeric computation. Dispersion relation between $\omega$ and $k$ can be symbolically represented by an implicit functional form $f(\omega, k)=0$. This relation may be explicitly solved in the form of real roots of $\omega=\psi(k)$. There are an infinite number of possible solutions, in general, which correspond to different wave modes. In case of plane waves, phase velocity vector is defined as $c_{p}=\left(\frac{\omega}{k}\right) \frac{\mathbf{k}}{|\mathbf{k}|}=\left(\frac{\omega}{k^{2}}\right) \mathbf{k}$. The vectors of velocity curves in the direction of a given $\mathbf{k}$ represent the admissible phase velocity dispersion of different wave modes. Dimensionless wave number $\left(k d / c_{T}\right)$ and dimensionless phase velocity $c_{p} / c_{T}$ employed to normalize the phase velocity, respectively. Furthermore, $c_{T}$ defined as $c_{T}=\sqrt{c_{66} / \rho}$ is the transverse (in-plane shear) wave velocity in the lamina. The material used in this study is orthotropic and are considered from Hawwa and Nayfeh (1995). Numerical examples demonstrate dispersion curves and characteristic curves in the lamina and laminate (Figures 1, 2, 3 and 4).

Distinction between mode types is quite artificial, when we study thermoelastic waves in anisotropic plates, as the equation for thermal and elastic wave modes, i.e., quasilongitudinal and quasi-transverse and shear horizontal modes, will generally be coupled with quasi-thermal modes. Also, for wave propagation in the direction of symmetry, some wave types revert to pure modes, leading to a simpler characteristic equation of lower order. A consequence of thermoelastic anisotropy in media is the loss of pure wave modes for general propagation direction. At low wave number limits, modes are found to highly influence and they vary with the thermal relaxation times. At relatively low values of the wave number, little change is seen in these values. As the wave number increases, other high modes appear; one of the modes seems to be associated with quick change in the slope of the mode. It is also observed that with changes in thermal relaxation times, lower modes have more influence whereas a small variation is noticed in the high modes. From these figures, it is observed that at low wave number limits, although wave speed modes are dispersive, but are different from the coupled case. Thus, in generalized thermoelasticity, at low values of the wave number, only the lower modes get affected and the little change is seen at relatively high values of the wave number. The low-value region of the wave number is found to be of more physical interest in generalized thermoelasticity. Further, since high wave number limits have no effect in generalized thermoelasticity, then the second sound effects are short lived. 


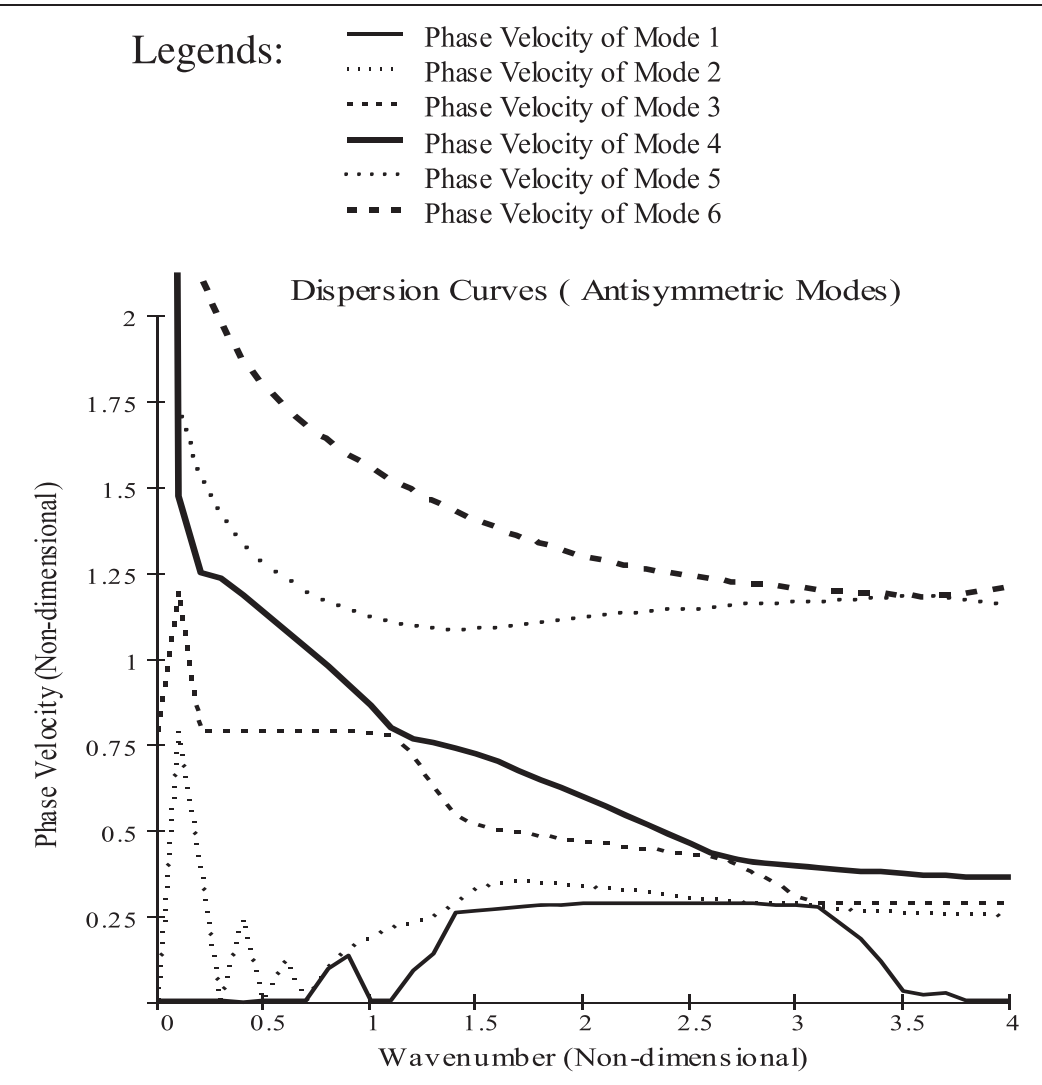

Figure 1 Dispersion curves of antisymmetric modes when thermal relaxation time $=2 \times 10^{-7} \mathrm{~s}$.

\section{Conclusions}

Propagation of guided thermoelastic waves in a homogeneous, orthotropic, thermally conducting plate is investigated within the framework of the generalized theory of thermoelasticity proposed by Lord and Shulman (1967).
This theory includes a thermal relaxation time in the heat conduction equation in order to model the finite speed of the thermal wave. The results show that both elastic and thermal modes are attenuated, the thermal modes exhibit much larger attenuation than the elastic modes. The

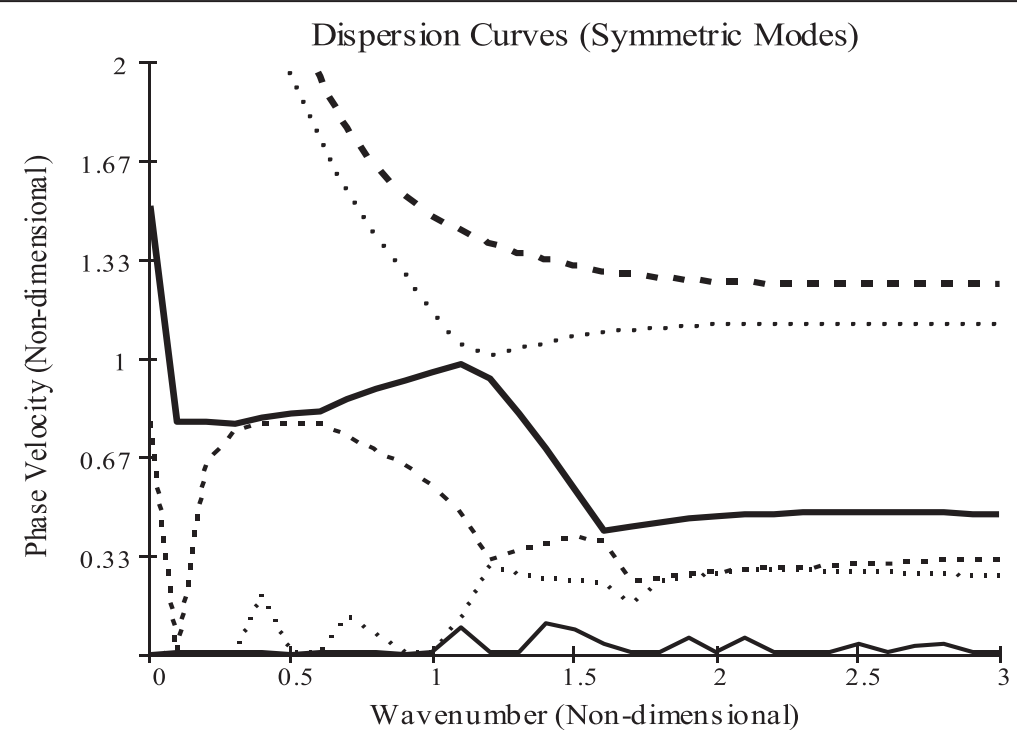

Figure 2 Dispersion curves of symmetric modes when thermal relaxation time $=2 \times 10^{-7} \mathrm{~s}$. 


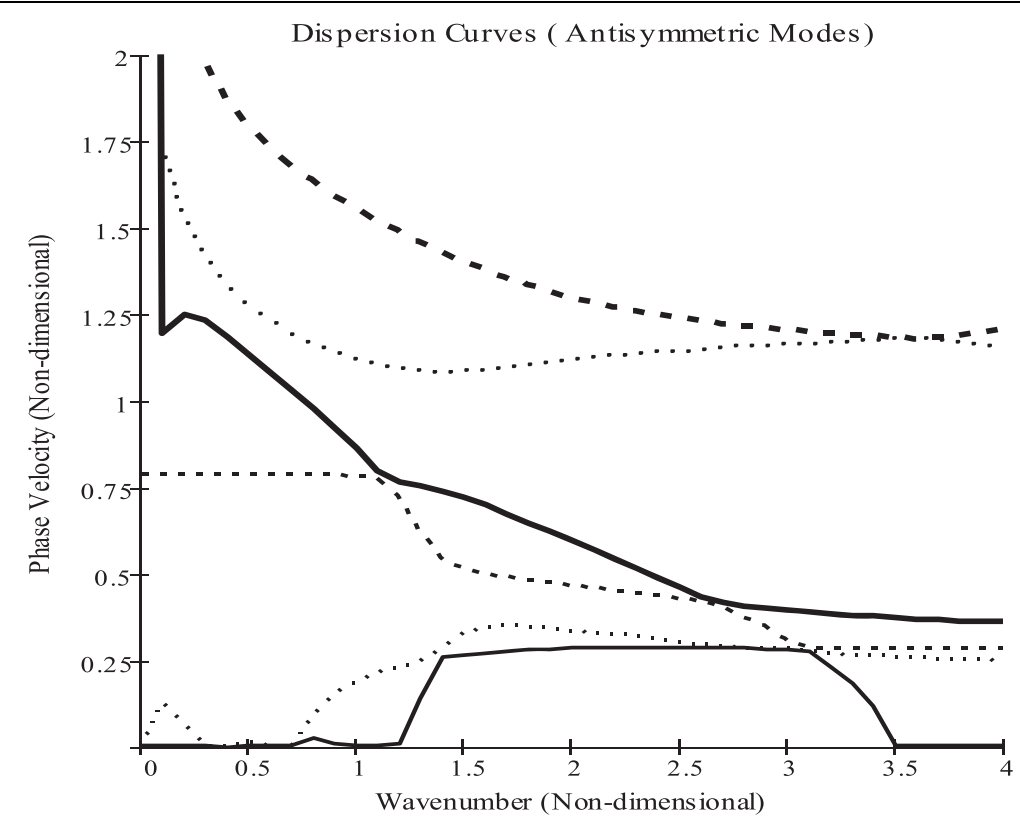

Figure 3 Dispersion curves of antisymmetric modes when thermal relaxation time $=2 \times 10^{-6} \mathrm{~s}$.

attenuation of the former is quite small. The results agree with previous observations by Hawwa and Nayfeh (1996). The coupling term is generally small for all materials and can be neglected. Neglecting the coupling term simplifies the analysis without noticeable effect on the frequency spectrum as we saw earlier. Because of the small relaxation time exhibited by the materials under consideration, the thermal wave modes have much larger phase speeds than the elastic modes. The effect of increasing the relaxation time is to lower the speeds of the thermal modes.
The effect of anisotropy of the material is quite pronounced on waves propagating in different directions along the plate. Thus, it is important to consider the anisotropy of the material in order to accurately model the propagation characteristics for material characterization and transient response. Employing a three-dimensional theory of thermoelasticity, solutions of thermoelastic Lamb waves in a lamina within the framework of the generalized theory of thermoelasticity proposed by Lord and Shulman (1967) are established, and obtained results are

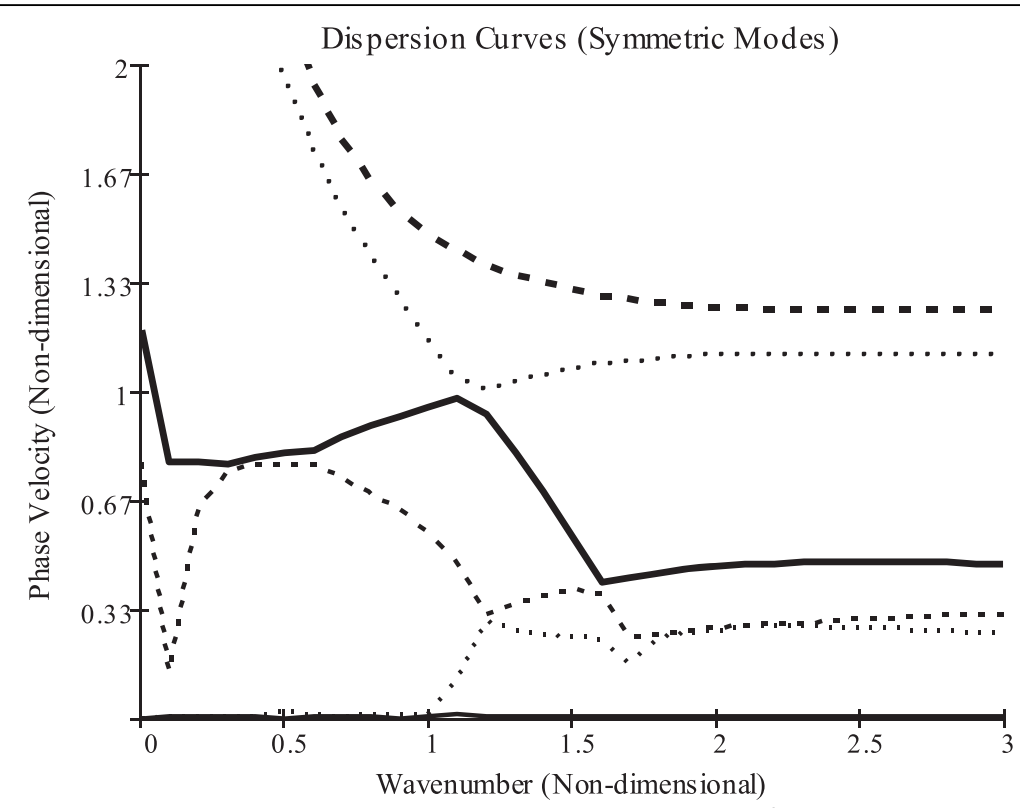

Figure 4 Dispersion curves of symmetric modes when thermal relaxation time $=2 \times 10^{-6} \mathrm{~s}$. 
extended to a general laminate with an arbitrary lay-up. Employing boundary conditions on both mid-plane and top surfaces is developed to decouple the wave modes for symmetric laminates.

\section{Competing interests}

The author declare that he has no significant competing financial, professional or personal interests that might have influenced the performance of the work described in this manuscript.

\section{Received: 2 March 2014 Accepted: 13 May 2014}

Published online: 07 August 2014

\section{References}

AN, A -e-n, \& Askar Nadia, A. (2014). Calculation of bulk acoustic wave propagation velocities in trigonal piezoelectric smart materials, applied mathematics \& information sciences. Sciences, 8(4), 1-8.

Abrate, S. (1998). Impact on composite structures. Cambridge: Cambridge University Press.

Achenbach, JD. (1973). Wave propagation in elastic solids. Amsterdam: North-Holland.

Banerjee, DK, \& Pao, YK. (1974). Thermoelastic waves in anisotropic solids. Journal of Acoustical Society of America, 56, 1444-1454.

Brekhovskikh, LM. (1960). Waves in layered media. New York: Academic Press.

Chadwick, P. (1960). Thermoelasticity: the Dynamic Theory. In R Hill \& IN Sneddon (Eds.), Progress in solid mechanics. North Holland, Amsterdam.

Chadwick, P. (1979). Basic properties of plane harmonic waves in a presented heat conducting elastic material. Journal of Thermal Stresses, 2, 193.

Chadwick, P, \& Seet, LTC. (1970). Wave propagation in transversely isotropic heat conducting elastic materials. Mathematica, 17, 225.

Chandrasekharaiah, DS. (1986). Thermoelasticity with second sound - A review. Applied Mechanics Review, 39, 355-376.

Chandrasekharaiah, DS. (1998). Hyperbolic thermoelasticity-a review of recent literature. Applied Mechanics Review, 51(12), 705-729.

Cementi, DE. (1997). Guided waves in plates and their use in materials characterizations. Applied Mechanics Review, 50(5), 247-283.

Choi, HY, \& Chang, FK. (1992). A model for predicting damage in graphite/epoxy laminated composites resulting from low-velocity point impact. Journal of Composite Materials, 26(14), 2134-2169.

Choi, HY, Downs, RJ, \& Chang, FK. (1991a). A new approach toward understanding damage mechanisms and mechanics of laminated composites due to low-velocity impact: Part I - experiments. Journal of Composite Materials, 25, 992-1011.

Choi, HY, Downs, RJ, \& Chang, FK. (1991b). A new approach toward understanding damage mechanisms and mechanics of laminated composites due to low-velocity imapct: Part II - analysis. Journal of Composite Materials, 25, 1012-1038.

Chawla, KK. (1987). Composite materials (pp. 189-191). New York: Springer-Verlag.

Dhaliwal, RS, \& Sherief, HH. (1980). Generalized thermoelasticity for anisotropic media. Quarterly Applied Mathematics, 38, 1-8.

Ewing, WM, Jardetzky, WS, \& Press, F. (1957). Elastic waves in layered media. New York: McGraw Hill.

Fukuda, H, Katoh, F, \& Yasuda, J. (1996). Low velocity impact damage of carbon fiber reinforced thermoplastics (pp. 139-145). Bakelma: Progress in Durability. Analysis of Composite Systems.

Green, AE, \& Lindsay, KA. (1972). Thermoelasticity. Journal of Elasticity, 2, 1-7.

Halpin, JC. (1992). Primer of composite material analysis (2nd ed., pp. 206-207). Lancaster: Technomic Publishing.

Hawwa, MA, \& Nayfeh, AH. (1996). Thermoelastic waves in laminated composites plate with a second sound effect. Journal of Appied Physics, 80, 2733-2738.

Hawwa, MA, \& Nayfeh, AH. (1995). The general problem of thermoelastic waves in anisotropic periodically laminated composites. Composite Engineering, 5, 1499-1517.

Hayhurst, CJ, Hiermaier, SJ, Clegg, RA, Riedel, W, \& Lambert, M. (1999). Development of material models for nextel and kevlar-epoxy for high pressures and strain rates (pp. 16-19). Huntsville, AL, Nov: Hypervelocity Impact Symposium.

Hiermaie, SJ, Riedel, W, Clegg, RA, Hayhurst, CJ, Wentzel, CM. (1999). Advanced material models for hypervelocity impact simulations AMMHIS. European Space Agency, Contract Report, EMI-Report br.E43/99, 30 July.
Jones, RM. (1975). Mechanics of composite materials. Washington: Scripta Book Co. Jones, RM. (1999). Mechanics of composite materials, Vol. 2-17 (2nd ed., pp. 190-221). New York: Taylor \& Francis.

Liu, GR, Tani, J, Watanabe, K, \& Ohyoshi, T. (1990). Lamb propagation waves in anisotropic laminate. ASME, Journal of Applied Mechanics, 57, 923-929.

Lord, HW, \& Shulman, Y. (1967). A generalized dynamical theory of thermoelasticity. Journal of Mechanics and physics of solids, 15, 299-309.

Nayfeh, AH. (1995). Wave propagation in layered anisotropic media with applications to composites. Amsterdam: Elsevier.

Nayfeh, AH, \& Chementi, DE. (1989). Free wave propagation in plates of general anisotropic media. Journal of Applied Mechanics, 56, 881-886.

Nayfeh, AH, \& Chementi, DE. (1991). General problem of elastic wave propagation in multilayered anisotropic media. Journal of Acoustical Society of America, 89, 1521-1531.

Nowacki, W. (1975). Dynamic problems of thermoelasticity. Leyden TheNetherlands: Noordho.

Nowacki, W. (1986). Thermoelasticity (2nd ed.). Oxford: Pergamon Press.

Thangjitham, S, \& Choi, HJ. (1991). Thermal stresses in a multilayered anisotropic medium. ASME, Journal of Applied Mechanics, 58(4), 1021-1027.

Tao, D, \& Prevost, JH. (1984). Relaxation effects on generalized thermoelastic waves. Journal of Thermal Stresses, 7, 79-89.

Vel, SS, \& Batra, RC. (2001). Generalized plane strain thermoelastic deformation of laminated anisotropic thick plates. International Journal of Solids and Structures, 38, 1395-1414.

Vel, SS, \& Batra, RC. (1999). Analytical solution for rectangular thick laminated plates subjected to arbitrary boundary conditions. AIAA Journal, 37, 1464-1473.

Vel, SS, \& Batra, RC. (2000). The generalized plane strain deformations of anisotropic composite laminated thick plates. International Journal of Solids and Structures, 37, 715-733.

Verma, KL, Hasebe, N, \& Sethuraman, R. (1999). Dynamic distribution of displacements and thermal stresses in multilayered media in generalized thermoelasticity. Proc. of third International Congress on Thermal Stresses, 577-580. Poland.

Verma, KL. (2001). Thermoelastic vibrations of transversely isotropic plate with thermal relaxations. International Journal of Solids and Structures, 38 $8529-8546$

Verma, KL, \& Hasebe, N. (2001). Wave propagation in plates of general anisotropic media in generalized thermoelasticity. International Journal of Engineering Science, 39(15), 1739-1763.

Verma, KL, \& Hasebe, N. (2002). Wave propagation in transversely isotropic plates in generalized thermoelasticity. Archive of Applied Mechanics, $72(6-7), 470-482$

Verma, KL. (2002). On the propagation of waves in layered anisotropic media in generalized thermoelasticity. International Journal of Engineering Science, 40(18), 2077-2096.

Verma, KL. (2012). On the thermoelastic waves in anisotropic laminated composite plates, Mechanics of Nano, Micro and Macro Composite Structures; Politecnico di Torino. 18-20 June 2012.

Wang, L, \& Yuan, FG. (2005). Damage identification in a composite plate using pre stack reverse-time migration technique. Structural Health Monitoring, 4(3), 195-211.

Wang, L, \& Yuan, FG. (2006). Experimental study of lamb wave propagation in composite laminates, smart structures and materials, sensors and smart structures technologies for civil, mechanical, and aerospace systems, edited by Masayoshi Tomizuka, et al. Proceedings of SPIE, 6174, 617442.

\section{doi:10.1186/s40712-014-0004-9}

Cite this article as: Verma: Thermoelastic symmetric and antisymmetric wave modes with trigonometric functions in laminated plates. International Journal of Mechanical and Materials Engineering 2014 1:4. 